\title{
Density and Structural Effects in the Radiation Tolerance of $\mathrm{TiO}_{2}$ Polymorphs
}

\author{
M. J. Qin ${ }^{1}$, E. Kuo ${ }^{1}$, K. R. Whittle ${ }^{1}$, S. C. Middleburgh ${ }^{1}$, \\ G. R. Lumpkin ${ }^{1}$, M. Robinson ${ }^{2}$, N. A. Marks ${ }^{2,3}$ \\ ${ }^{1}$ Institute of Materials Engineering, Australian Nuclear Science and Technology \\ Organisation, Locked Bag 2001, Kirrawee DC, NSW 2232, Australia. \\ ${ }^{2}$ Nanochemistry Research Institute, Curtin University, PO Box U1987, Perth \\ WA 6845, Australia. \\ ${ }^{3}$ Discipline of Physics \& Astronomy, Curtin University, PO Box U1987, Perth \\ WA 6845 , Australia. \\ E-mail: mqi@ansto.gov.au
}

\begin{abstract}
The radiation response of $\mathrm{TiO}_{2}$ has been studied using molecular dynamics. The simulations are motivated by experimental observations that the three low-pressure polymorphs, rutile, brookite and anatase, exhibit vastly different tolerance to amorphization under ion-beam irradiation. To understand the role of structure we perform large numbers of simulations using the smal thermal spike method. We quantify to high statistical accuracy the number of defects created as a function of temperature and structure type, and reproduce all the main trends observed experimentally. To evaluate a hypothesis that volumetric strain relative to the amorphous phase is an important driving force for defect recovery we perform spike simulations in which the crystalline density is varied over a wide range. Remarkably, the large differences between the polymorphs disappear once the density difference is taken into account. This finding demonstrates that density is an important factor which controls radiation tolerance in $\mathrm{TiO}_{2}$.
\end{abstract}

PACS numbers: 61.43.Bn; 61.80.Jh; 61.82.Ms 


\section{Introduction}

Due to their potential applications in advanced reactors and nuclear waste forms, radiation tolerant crystalline ceramics have been the subject of intensive investigations, with the aim to understand radiation induced phenomena, especially amorphization and recrystallization $[1,2,3,4,5,6,7]$. However, the physical process governing the energetics of defect production, migration, and recombination are not well understood for many materials. Recently the effects of structure, bonding, and the ability to accommodate lattice disorder have been proposed as important factors affecting the radiation induced amorphization in ceramics $[3,6,7]$. For example, by performing radiation-induced amorphization experiments on fluorite-related $\mathrm{MO}_{2}$ structures, Sickafus et al. $[6,7]$ proposed that the radiation damage recovery in these materials is related to the ability to accommodate lattice disorder.

The various polymorphs of $\mathrm{TiO}_{2}$ provide an instructive point of comparison to the fluorite-type class of structures. Large differences in radiation response have been observed [4,5] amongst the $\mathrm{TiO}_{2}$ polymorphs, but this behaviour is not consistent with a lattice disorder mechanism as the titania structures have no inherent disorder pathway, i.e. cation antisite and anion-vacancy disorder are not possible. This situation implies that additional fundamental properties of the structure influence defect formation, migration, and ultimately the ability to accommodate amorphous domains. In order to investigate this structure effect, we recently performed ion irradiation experiments $[4,5]$ on the three common polymorphs of $\mathrm{TiO}_{2}$ : rutile, brookite and anatase. As all three phases have the same chemical composition and similar covalence, the effect of structure on the radiation-induced amorphization and recrystallization can be investigated. The experiments revealed the best radiation tolerance in rutile, the worst in anatase, and intermediate behaviour in brookite. In the case of synthetically prepared rutile, the radiation resistance was exceptional; even at temperatures of just $50 \mathrm{~K}$ the structure remained crystalline at fluences up to $5 \times 10^{15}$ ions $/ \mathrm{cm}^{2}$. One suggestion arising from the experiments is that the radiation resistance is related to the degree of polyhedral distortion and density of the three polymorphs. In this work we explore this hypothesis using atomistic simulation.

Recently we have performed [4, 8] molecular dynamics simulations on the three polymorphs of $\mathrm{TiO}_{2}$. Instead of carrying out traditional large-scale cascade simulations $[9,10,11]$, we adopted the spherical thermal spike technique [12]. Computational cost-effectiveness is one of the benefits of using small spike simulations, although it has been pointed out by Crocombette [13] that quantitatively thermal spikes generate fewer Frenkel pairs than collision cascades. Nevertheless, the initial thermal spikes simulations qualitatively reproduced most of the important features of the experiments, in particular the observation that radiation resistance was highest for rutile and lowest for anatase.

In this work, we extend upon our initial work by strengthening the methodology and using this improved approach to test the hypothesis that volume-based strain is a key factor in the radiation response of the $\mathrm{TiO}_{2}$ polymorphs. The improved technical aspects of the simulations relate to lattice equilibration at finite temperature, edge thermostats to remove heat and large numbers of simulations for the collection of good quality statistics. The strain hypothesis is tested in a novel way by simulating radiation response in materials under negative hydrostatic pressure. These "virtual experiments" do not have have a natural laboratory analogue and illustrate the strength of computation as a tool to probe the atomistic origins of radiation resistance. 


\section{Computational Details}

All calculations were performed using the empirical potential developed by Matsui and Akaogi (MA) [14] for $\mathrm{TiO}_{2}$. The MA potential is a Buckingham-type model employing partial charges $\left(\mathrm{O}^{-1.098}\right.$ and $\left.\mathrm{Ti}^{+2.196}\right)$ and has been successfully used to predict the structure and properties of a range of $\mathrm{TiO}_{2}$ polymorphs $[15,16]$. Since simulations of radiation damage involve atomic configurations far from equilibrium, the MA potential is splined at short range to a Ziegler-Biersack-Littmark (ZBL) screened Coulomb potential [17] using an exponential function [18, 19].

The molecular dynamics (MD) simulations were performed using the DL_POLY 3 simulation package [20]. The velocity Verlet integrator was used to update the atomic positions and velocities and all calculations used periodic boundary conditions and a variable timestep initially set to $1 \mathrm{fs}$. Coulombic interactions were calculated using Ewald summation and a cutoff of $10 \AA$ was used for the short range interactions. The calculations were performed within the NVE ensemble suplemented by a Langevin wall thermostat with a $3 \AA$ thickness [20]. The temperature of the wall thermostat was set to be same as that of the equilibrated lattice prior to the introduction of the thermal spike. The inclusion of the wall thermostat and the finite temperature of the initial configuration improves on the methodology employed in our earlier work [8] in which wall thermostats were not used and the initial temperature was zero Kelvin. This approach also avoids unphysical temperature increases associated with the introduction of thermal spikes, which in some instances can be of concern $[21,22,23]$. The structures of rutile [24], brookite [24] and anatase [25] were optimised using the General Utility Lattice Program (GULP) [26] and subsequently used in all MD simulations. GULP was also used to create rutile and brookite structures under negative and positive pressure in order to test the hypothesis of volume-based strain.

The thermal spike methodology follows our earlier work [8] which was in turn inspired by Santos et al. [12]. The majority of the simulations employed $16 \times 16 \times 24$ supercells $(36,864$ atoms $)$ of rutile, $8 \times 14 \times 14$ supercells $(37,632$ atoms $)$ of brookite, and $20 \times 20 \times 8$ supercells $(38,400$ atoms $)$ of anatase. In all cases the lattices were first equilibrated at temperatures spanning 50-550 K. After equilibration, 137 atoms within a sphere of radius about $7 \AA$ were identified for the purpose of initiating the thermal spike, with the precise value of the radius varying slightly depending on the density of the structure. All atoms inside the sphere were subsequently assigned a kinetic energy of $5 \mathrm{eV}$ in a randomly selected direction. This energy is sufficient to rapidly induce transient melting within the spike. An example of this liquid-like state is shown for brookite in figure 1 where after just 0.13 ps the radial distribution function in the region $5 \AA$ from the centre of the spike closely approximates that of liquid $\mathrm{TiO}_{2}$ at $3500 \mathrm{~K}$. The data for liquid $\mathrm{TiO}_{2}$ was calculated using the same simulation box as employed for the thermal spike; the liquid state was initiated by randomly displacing atoms by up to $0.4 \AA$ and subsequently equilibrating at $3500 \mathrm{~K}$ for 90 ps.

The total kinetic energy introduced into the supercell was very close to $677 \mathrm{eV}$ with the precise value varying by up to $1 \mathrm{eV}$ due to the requirement of zero linear momentum. The time evolution of the thermal spikes was then followed for at least 20 ps. Defect generation was quantified by comparing the instantaneous state of the system to an initial reference structure, using a distance-based criterion of $0.6 \AA$ to identify when an atom was not on a lattice site. To enable the collection of reliable statistics a large number of spike simulations (typically 100) were performed for each combination of computational conditions, using randomly selected initial directions 

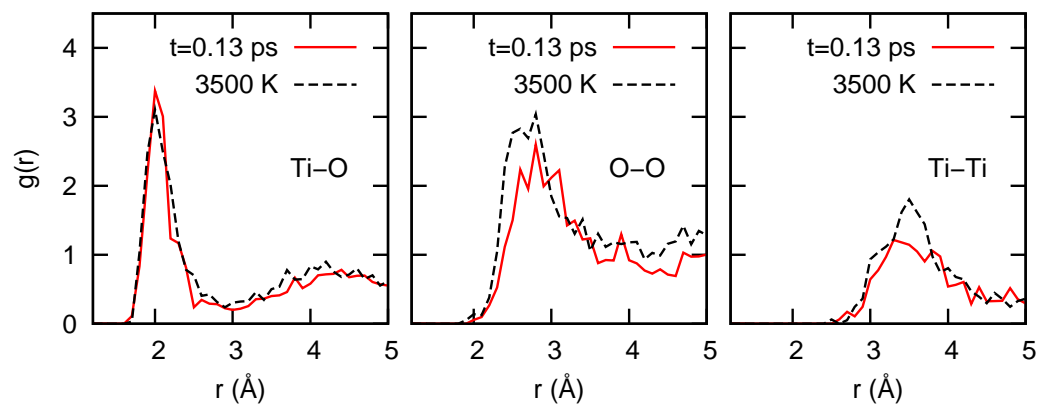

Figure 1. Partial radial distribution functions, $g(r)$, for a thermal spike created in brookite initially at $50 \mathrm{~K}$. Red lines indicate the system at $\mathrm{t}=0.13 \mathrm{ps}$. Black lines indicate additional simulation results for bulk liquid $\mathrm{TiO}_{2}$ at $3500 \mathrm{~K}$. For both the spike and the liquid an analysis volume of radius $5 \AA$ was employed, and hence $\mathrm{g}(\mathrm{r})$ does not tend to unity at large $\mathrm{r}$. This approach enables a direct comparison between the spike and bulk liquid.

for the spike atoms.

For verification purposes, we performed a series of small-scale cascade simulations in rutile using the same supercells as employed for the thermal spikes. The cascade was initiated from a Ti primary knock-on atom (PKA) having the same total kinetic energy as used in the thermal spike calculations, that is, $677 \mathrm{eV}$. For comparison, we also carried out a small number of large-scale cascade calculations in rutile $(576,000$ atoms), brookite (511,632 atoms) and anatase (525,312 atoms) supercells with a $5 \mathrm{keV}$ Ti PKA.

\section{Results and Discussion}

\subsection{The Effect of Structure on Defect Recovery}

Using the framework described above, thermal spikes were generated in rutile, brookite and anatase for temperatures between 50 and $550 \mathrm{~K}$. In order to accurately capture a statistical sense of the effect of structure, 100 simulations were performed for each combination of temperature and polymorph. An example of the importance of statistical sampling is provided in figure 2 which shows two simulations in brookite for an initial (i.e. pre-spike) temperature of $50 \mathrm{~K}$. In all respects the simulations are identical with respect to the initial positions of the atoms and the amount of kinetic energy added to the system; the only factor which is different is the direction of the atomic velocities in the spike region itself. In one instance the transient damage created by the spike is completely absence after several picoseconds, while in the other case an amorphous pocket remains. Capturing this variability is an important improvement over earlier work where only a handful of simulations were performed across the various conditions.

The extent of the inherent statistical variability is captured quantitatively in figure 3 which shows data for 100 simulations in brookite at an initial temperature of $50 \mathrm{~K}$. The figure shows the number of defects in the simulation cell as a function of time, with each gray line describing an individual simulation. As noted above, each simulation uses the same spike parameters but with different initial (random) velocity 

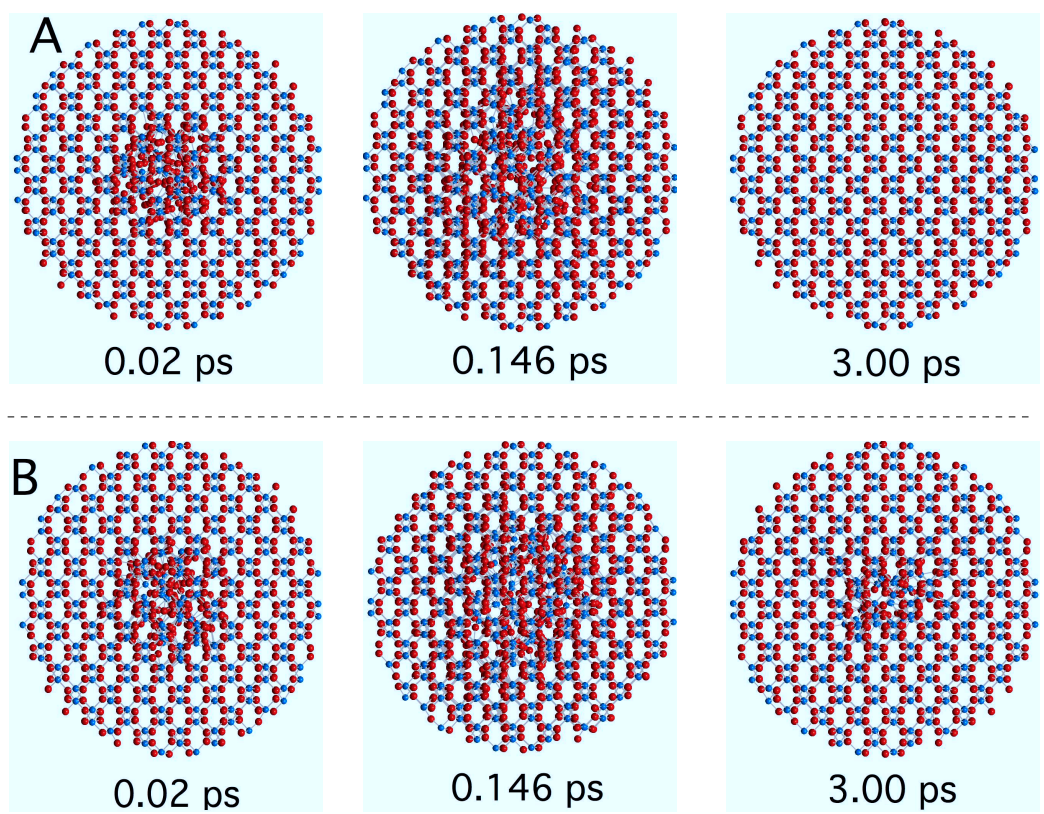

Figure 2. Time evolution of two spherical thermal spikes in brookite with a pre-spike temperature of $50 \mathrm{~K}$. Ti and $\mathrm{O}$ atoms are shown as light-blue and red spheres, respectively. Only atoms within a radius of $20 \AA$ of the spike centre are displayed. Both simulations employ the same initial coordinates but different initial velocity directions for the spike atoms. Simulation A exhibits full radiation damage recovery, while simulation B shows only partial recovery.

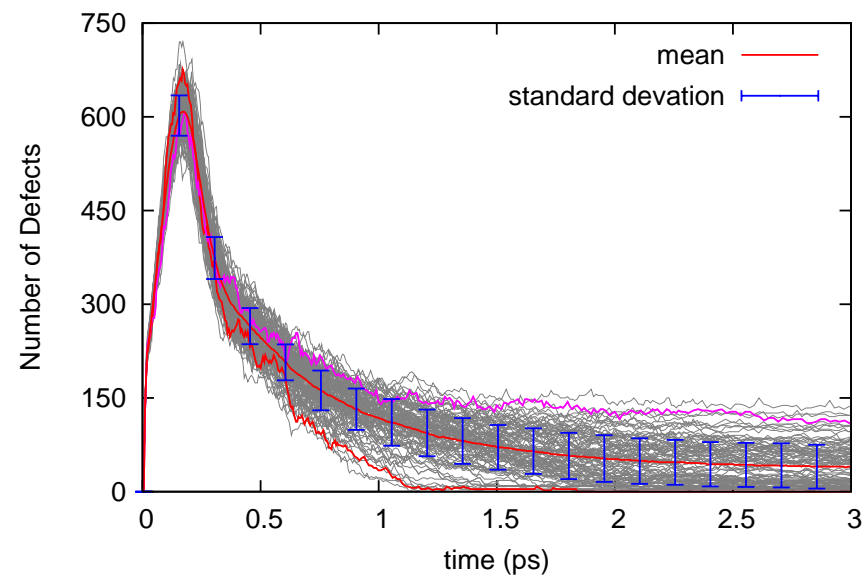

Figure 3. Number of defects as a function of time for thermal spikes in brookite. The initial pre-spike temperature is $50 \mathrm{~K}$. Gray lines indicate results for 100 different simulations employing randomly chosen initial velocity directions for the spike atoms. The blue line and blue bars indicate the mean and one standard deviation, respectively. The red and purple solid lines correspond to simulations $\mathrm{A}$ and $\mathrm{B}$ in figure 2, respectively. 
directions for the selected atoms. The blue line is the average of all the curves and the blue bars indicate one standard deviation. The solid red and purple lines correspond to the specific cases seen in figure 2 , showing that neither is particularly representative of the statistical average for brookite under these conditions.

Having captured the statistical spread it is possible to quantify the properties of a spike in brookite at $50 \mathrm{~K}$. Regardless of the data set, a maximum number of defects is observed around 0.17 ps after initiation, with typically around 600 defects being generated. After this time the number of defects rapidly decreases as the surrounding crystalline region acts both as a heat sink to cool the spike as well as a template that nucleates recrystallisation. While a small number of simulations (15 out of 100$)$ showed complete recovery, on average a total of 37 defects remained at the conclusion of the spike, with some spikes having as many as 135 defects. In the context of radiation damage due to ion beams or other energetic sources, these residual defects are the fundamental entity which gradually accumulate to the point that the entire structure is rendered amorphous. We note that the time corresponding to the maximum number of defects is very similar to the value of 0.14 ps reported in Ref. [8] (see table I) which used a smaller spike radius of $5 \AA$ and a higher kinetic energy per atom of $6 \mathrm{eV}$. This demonstrates that the general behaviour of the spikes are relatively insensitive to the parameters employed, subject to the caveat that the energy and radius should be neither excessively large nor small. Further work involving a sensitivity analysis of the spike to these parameters would be a natural extension of this study.

Figure 4 compares the defect evolution with time in anatase, brookite, and rutile for an initial temperature of $50 \mathrm{~K}$. As seen previously [8], defect generation and recovery are very different across the three polymorphs. The significant advance here concerns the statistical reliabilty of the new results and the degree of confidence that can be placed in the number of defects produced. Figure 4 shows there is much less disorder produced in rutile (with a maximum number of defects of $\sim 500$ ), much more disorder in anatase (maximum number of defects of $\sim 900$ ), and brookite shows intermediate behavior (about 600 defects). Less time is required for rutile to reach the maximum defect population (0.145 ps) compared to anatase $(0.25 \mathrm{ps})$ and brookite

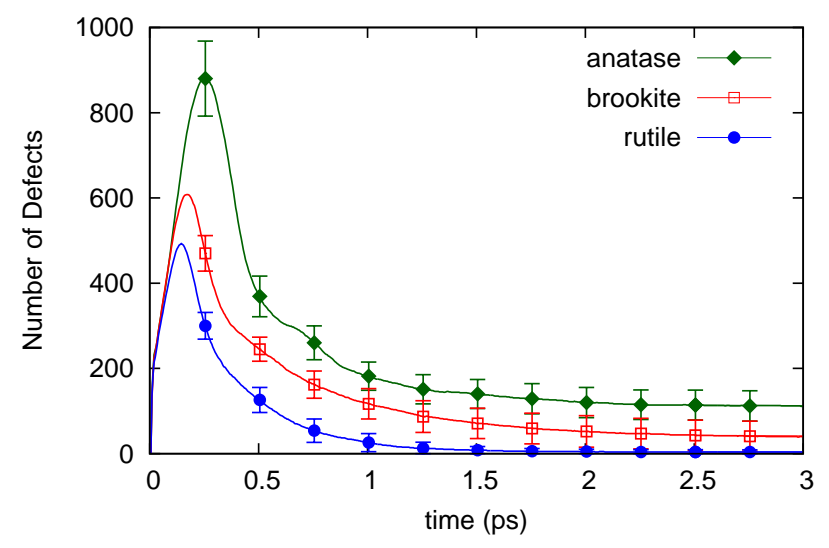

Figure 4. Time evolution of the number of defects produced by thermal spikes in rutile (blue circles), brookite (red squares) and anatase (green diamonds). Points and bars indicate the mean and one standard deviation, respectively, computed from 100 simulations. All calculations used a pre-spike temperature of $50 \mathrm{~K}$. 
(0.17 ps). After $1.5 \mathrm{ps}$, much of the disorder in rutile is recovered (on average, only 4 defects remain). In contrast, considerable disorder is still present for both brookite (about 37 defects) and anatase (about 110 defects), even after 3 ps.

Simulations extending up to 20 ps were performed for all polymorphs and little further change was observed. Generally speaking, rutile often showed complete recovery (52 instances out of 100 simulations), anatase always contained defects, while in brookite only some simulations (15 out of 100) fully recovered. This behaviour is in excellent agreement with experimental studies $[4,5]$ which demonstrate a strong structural effect. In particular, the experimental trend of damage following the pattern rutile $<$ brookite $<$ anatase is reproduced in all aspects by the simulations, with rutile showing the smallest and shortest spike as well as the fewest surviving defects. Again, in direct alignment with experiment, brookite shows intermediate behaviour for all three quantities, while each quantity is largest for anatase.

One potential deficiency of earlier work was that temperature was not explicitly controlled. Here, the lattice is equilibrated at a prescribed (and finite) temperature and excess heat is removed via wall thermostats set to the same value. As previously noted [8], neglecting such details is not always a major factor, particularly if the number of atoms is sufficiently large to absorb the spike. Regardless, it is preferable to address these matters in the strictest possible sense, particularly when quantitative insight is sought. Against this background, figure 5 shows the effect of temperature on two of the most important spike quantities, namely the maximum and final number of defects. As anticipated, the effect of temperature on the behaviour of the spike is modest, with no changes evident regarding the relative ordering amongst the various

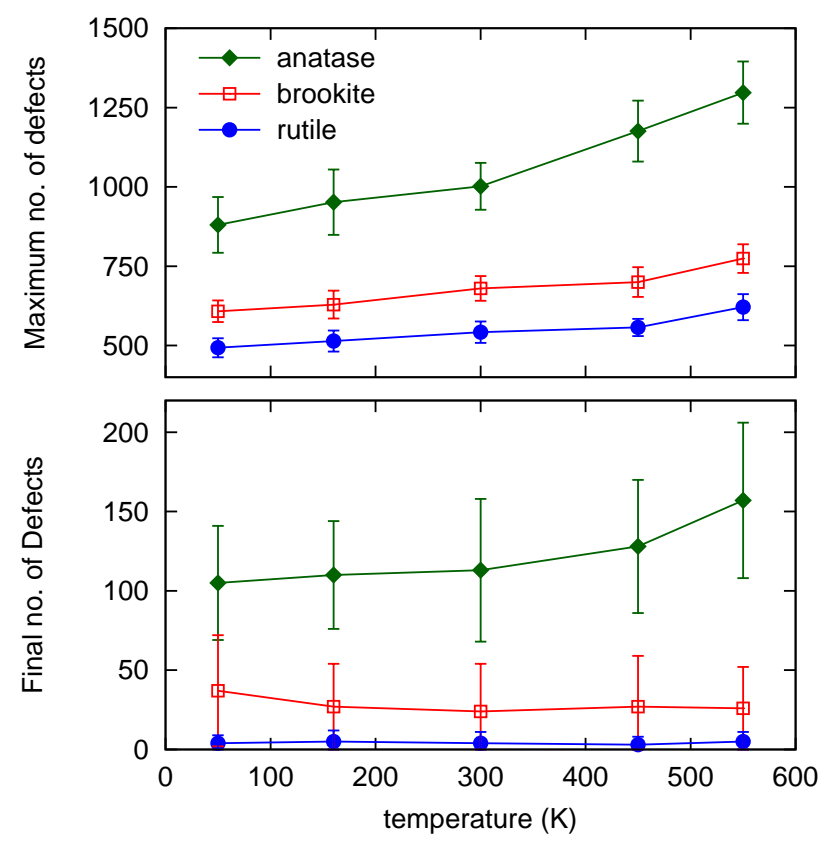

Figure 5. Effect of temperature on the maximum and final number of defects in spherical thermal spikes in rutile (blue circles), brookite (red squares) and anatase (green diamonds). 
polymorphs. The most significant trend with temperature is a slight increase in the maximum number of defects, indicating that during the ballistic phase a higher temperature assists the generation of disorder. The final number of defects shows a much weaker temperature effect, since elevated temperature assists with thermally activation recombination, even on this comparitively short timescale. This behaviour is reminiscent of a a similar effect seen in recent calculations of threshold displacement energies in rutile as a function of temperature [27].

\subsection{The Effect of Density on Defect Recovery}

The large differences amongst the $\mathrm{TiO}_{2}$ polymorphs prompts the consideration of an underlying explanation. As noted previously [8], the observed trends lie in contrast with structural freedom arguments [28, 29] based on edge sharing which predict that rutile is the least radiation tolerant polymorph and antase is the most tolerant. An important concept when considering $\mathrm{TiO}_{2}$ polymorphs are volumetric trends in relation to octahedral distortion and the number of shared edges. Somewhat counterintuitively, the number of shared edges between $\mathrm{TiO}_{6}$ octahedra (2 in rutile, 3 in brookite and 4 in anatase) correlates inversely to density, with rutile having the smallest molar volume and anatase the highest. As explained in Ref. [4], this behaviour is a consequence of increasing octahedral distortion across the series rutile to brookite to anatase. Another key idea in Ref. [4] is a hypothesis that the differing radiation tolerance amongst the $\mathrm{TiO}_{2}$ polymorphs is due to volumetric strain between the amorphous and crystalline phases. This argument is based on two assumptions: (i) that amorphous $\mathrm{TiO}_{2}$ produced by irradiation is the same for all three polymorphs, and (ii) that the density of amorphous $\mathrm{TiO}_{2}$ is $3.80 \mathrm{~g} / \mathrm{cm}^{3}$ as reported by Hoang [30]. Coupled to these assumptions is the observation that the experimental densities of rutile, brookite and anatase are 4.24, 4.13 and $3.90 \mathrm{~g} / \mathrm{cm}^{3}$, respectively. Accordingly, an amorphous pocket within rutile will be highly strained (i.e. compressed by the surroundings), while in anatase it will be only moderately strained. This volume difference between the crystalline and amorphous phases $\left(\Delta \mathrm{V}_{c a}\right)$ is approximately $12 \%$ for rutile, $9 \%$ for brookite and $3 \%$ for anatase. Since these three quantities correlate with the radiation response seen in experiments and simulation it was proposed that the volume strain associated with amporphous domains might contribute an important driving force for the recovery of defects and hence provide a possible explanation for the observed behaviour.

To test the strain hypothesis we performed a set of thermal spike simulations in which rutile and brookite structures were isotropically stretched and compressed. If our hypothesis is correct, then we would expect that similar radiation response would be observed under conditions when all three structures have the same density. Properties of a selection of stretched and compressed structures are shown in table I, along with their zero pressure equivalents. Varying the density is straighforwardly achieved using the GULP software; by applying either a positive or negative pressure the density can be decreased or increased to any desired value. The cell parameters determined from GULP were left unchanged in the subsequent spike simulations performed with DL_POLY. We did not perform any simulations of anatase under positive pressure as it transforms to other phases $\left(\mathrm{TiO}_{2}\right.$-II and rutile) at pressures between zero and $18 \mathrm{GPa}$, depending on temperature [31]. The table lists the important quantities needed to test the volume-strain hypothesis, namely the applied pressure, the resultant density and the molar volume change $\left(\Delta \mathrm{V}_{c a}\right)$ relative to 


\begin{tabular}{lcccc}
\hline Structure & $\begin{array}{c}\text { Pressure } \\
(\mathrm{GPa})\end{array}$ & $\begin{array}{c}\text { Density } \\
\left(\mathrm{g} / \mathrm{cm}^{3}\right)\end{array}$ & $\begin{array}{c}\Delta \mathrm{V}_{c a} \\
(\%)\end{array}$ & $\begin{array}{c}\text { Quadratic } \\
\text { Elongation }\end{array}$ \\
\hline Anatase & 0 & 3.90 & 2.7 & 1.031 \\
\hline \multirow{2}{*}{ Brookite } & -11.9 & 3.90 & 2.7 & 1.022 \\
& 0 & 4.18 & 10.1 & 1.020 \\
& 5.2 & 4.29 & 12.9 & 1.019 \\
\hline \multirow{3}{*}{ Rutile } & -19.7 & 3.90 & 2.7 & 1.013 \\
& -17 & 3.98 & 4.7 & 1.012 \\
& -10 & 4.16 & 9.6 & 1.011 \\
& 0 & 4.37 & 15.0 & 1.012 \\
& 5.2 & 4.46 & 17.4 & 1.011 \\
& 11 & 4.55 & 19.8 & 1.011 \\
\hline
\end{tabular}

Table 1. Structural parameters in three $\mathrm{TiO}_{2}$ polymorphs as a function of applied pressure. The molar volume change $\left(\Delta V_{c a}\right)$ quantifies the difference between the indicated crystalline structure and an amorphous phase at $3.80 \mathrm{~g} / \mathrm{cm}^{3}$. The quadratic elongation provides a measure of octahedral distortion as described in the text.

amorphous $\mathrm{TiO}_{2}$ at $3.80 \mathrm{~g} / \mathrm{cm}^{3}$. Note that while the molar volume change has the same trend as per experiment, the precise values differ slightly since equilibrium densities according to the MA potential are employed. Also shown in the table is the quadratic elongation $\langle\lambda\rangle_{\text {oct }}$ which quantifies the degree of distortion of a polyhedron from a perfect polyhedron of the same volume. The definition follows Ref. [4], namely,

$$
<\lambda>=\sum_{i=1}^{n}\left(d_{i} / d_{0}\right)^{2} / n
$$

where $d_{0}$ is the bond length of the corresponding perfect polyhedron and $n$ is the total number of $\mathrm{Ti}-\mathrm{O}$ bond length and $\mathrm{O}-\mathrm{O}$ edge lengths.

Table I shows that under conditions of large negative pressure $(-19.7 \mathrm{GPa})$ rutile has the same density as anatase, while for for brookite only $-11.9 \mathrm{GPa}$ is required as its natural density is closer to that of anatase. At these pressures the molar volume change relative to the amorphous phase is $2.7 \%$. The critical insight from the table is that at higher densities the molar volume change becomes increasing larger as noted above, while the quadratic elongation is essentially unaffected by the imposition of pressure. The latter observation shows that the topology of the structures remains much the same, even when the density is far from the zero-pressure value.

The effect of changing the density is illustrated in figure 6 which shows the number of defects as a function of time for thermal spikes in rutile at $50 \mathrm{~K}$. The calculation at $4.37 \mathrm{~g} / \mathrm{cm}^{3}$ has zero pressure, this being the equilibrium density for rutile in the MA potential. All other densities are under negative pressure according to table 1 . Reducing the density has a substantial effect, with lower densities generating many more defects at all stages during the evolution of the spike. The maximum number of defects in the $3.90 \mathrm{~g} / \mathrm{cm}^{3}$ simulation is more than three times higher than for the zero pressure case, and the final structure contains a large number of defects, forming an amorphous pocket. This observation provides immediate support for the volume-strain hypothesis, since the less dense rutile structures exhibit far less radiation resistance than at zero pressure.

Further support for the strain hypothesis comes from figure 7 which shows data for all three polymorphs at similar densities. Here the rutile structure $\left(\rho=3.98 \mathrm{~g} / \mathrm{cm}^{3}\right.$ and $\mathrm{P}=-17 \mathrm{GPa})$ and brookite structure $\left(\rho=3.90 \mathrm{~g} / \mathrm{cm}^{3}\right.$ and $\left.\mathrm{P}=-11.9 \mathrm{GPa}\right)$ have very similar defect evolution behaviour to anatase at its equilibrium density of $3.90 \mathrm{~g} / \mathrm{cm}^{3}$. 


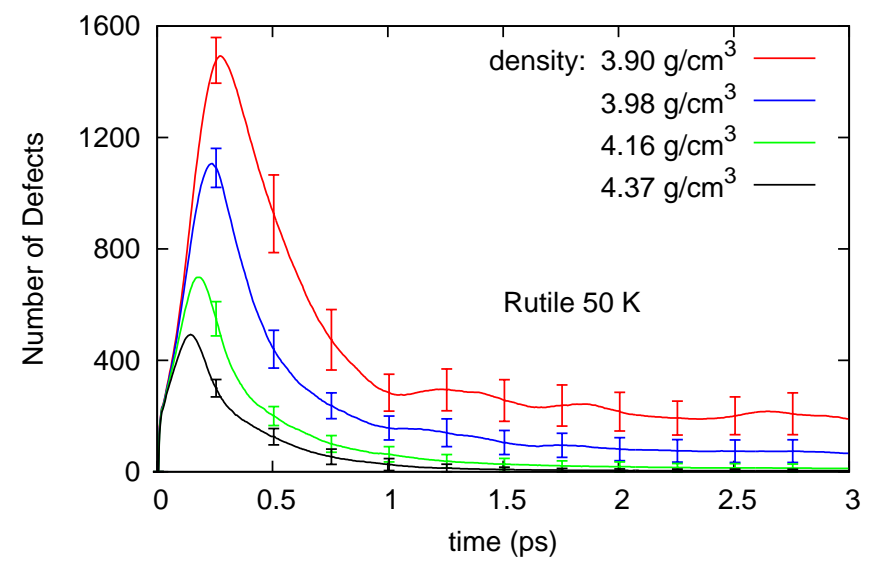

Figure 6. Time evolution of defects in spherical thermal spikes in rutile as a function of density. The zero pressure density is $4.37 \mathrm{~g} / \mathrm{cm}^{3}$, while all other densities correspond to negative pressure as per table 1. All calculations had an initial temperature of $50 \mathrm{~K}$.

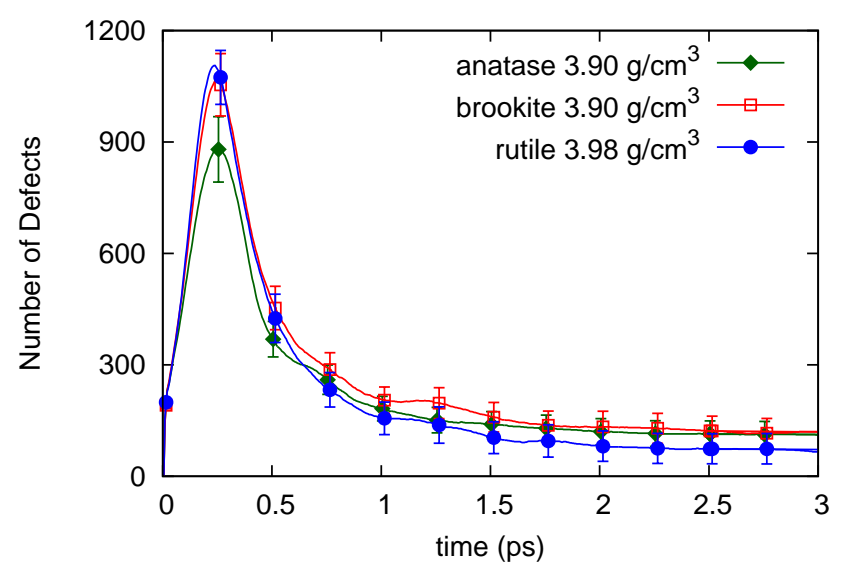

Figure 7. Time evolution of defects in spherical thermal spikes in rutile, brookite and anatase with similar densities. Rutile and brookite are both under negative pressure, while anatase is not. All calculations had an initial temperature of $50 \mathrm{~K}$.

Across all three polymorphs the maximum and final number of defects are remarkably similar. This behaviour provides deep insight into the radiation response mechanism which traditionally is considered from the viewpoint of defect energetics and migration barriers for recombination. In figure 7 we see clear evidence that a quantity as simple as volume (or density) can help explain why one structure is radiation resistant while another is not. This finding demonstrates that the radiation resistance of $\mathrm{TiO}_{2}$ can be fundamentally attributed to the respective densities of the polymorphs, rather than a more complex structual property.

To quantify the density effect we performed further calculations using compressed brookite. Figure 8 shows data for brookite and rutile at a number of densities as well as anatase at its equilibrium density. Rutile and brookite show similar trends in relation 

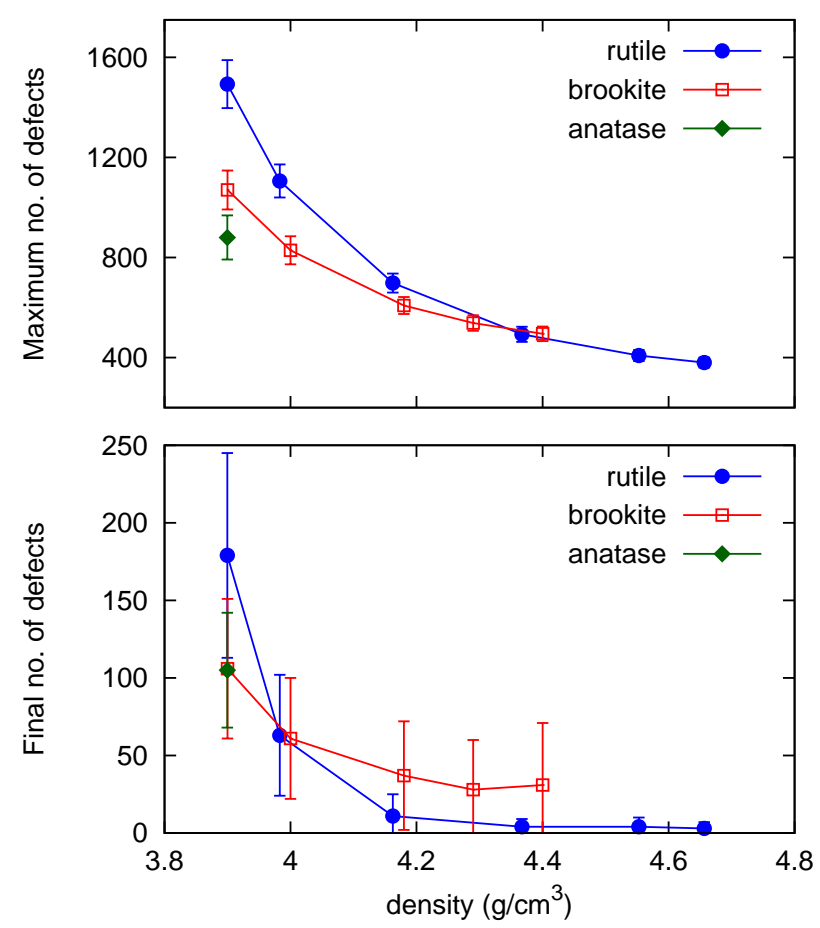

Figure 8. Maximum and final number of defects in thermal spikes in rutile, brookite and anatase as a function of density. Error bars indicate one standard deviation and the initial temperature was $50 \mathrm{~K}$.

to the maximum and final number of defects, with higher densities suppressing both the number of defects created and the number remaining once the spike has cooled. However, density is clearly not the only factor, as the two data sets are not identical. For example, at high density the final number of defects in rutile approaches zero, whereas in brookite it plateaus around 30. Accordingly, there must be additional (and, as yet, unknown) structural effects which play a second-order effect in defect generation and recovery. The primary influence however, is clearly the density, a phenomenomon which has previously only been speculated upon and may well have considerable utility in other materials systems.

\subsection{Cascade Simulations}

It has been noted in studies by Crocombette [13] that thermal spike simulations generally create fewer Frenkel pairs than cascades. Those studies used cylindrical thermal spikes designed from a cascade imparting an equivalent energy and of comparable size. As spherical thermal spikes are used in this paper, we performed a number of small-scale displacement cascade simulations in rutile, using a $677 \mathrm{keV}$ Ti PKA (this being the same energy as the thermal spikes) and 100 randomly selected initial directions. The number of defects as a function of time, averaged over all PKA directions, are shown as a blue line in figure $9(\mathrm{a})$. The red line in the same figure indicates the average number defects from thermal spikes simulations in rutile. On 


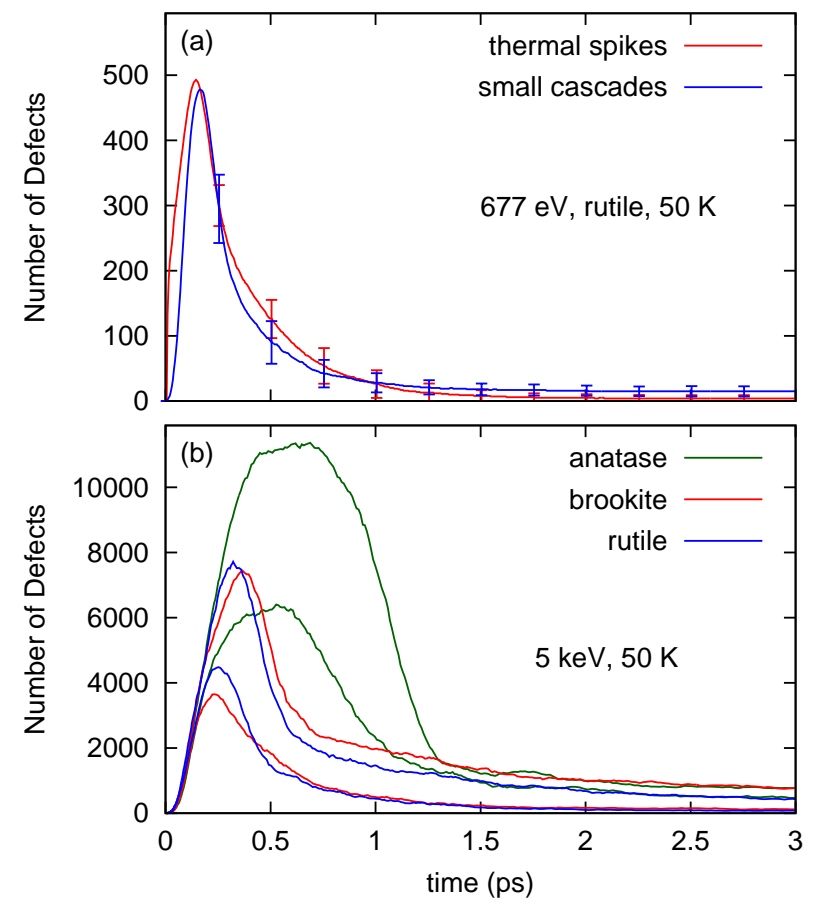

Figure 9. (a) Number of defects as a function of time in spherical thermal spikes (red line) and small cascades (blue line). All calculations were performed using a rutile lattice initially at $50 \mathrm{~K}$. The thermal spikes used the same conditions as elsewhere in this work, and the cascades were initiated with a PKA of a equivalent energy (i.e. $677 \mathrm{eV}$ ). One hundred simulations were performed for each data set; the error bars indicate one standard deviation. (b) Number of defects as a function of time in large-scale cascades initiated by a $5 \mathrm{keV}$ Ti PKA in rutile (blue), brookite (red) and anatase (green) lattices initially at $50 \mathrm{~K}$. Data from two simulations in each polymorph are shown.

average, the cascade simulations create slightly fewer defects at the peak than thermal spikes ( $516 \pm 30$ for thermal spikes versus $476 \pm 47$ for cascades). However, after recovery, more defects remain in the cascade simulations $(13 \pm 7)$ as compared to the thermal spikes $(3 \pm 5)$. In general, however, the trends from both the small cascade simulations and from thermal spikes simulations are very similar, demonstrating that the spherical thermal spikes provide a good representation of kinetic energy delivery into a small volume.

For illustration, we also performed a small number of large-scale cascade simulations in rutile, brookite and anatase using a $5 \mathrm{keV}$ Ti PKA in various offaxis directions. The time-evolution of the number of defects for two simulations in each polymorph are shown in figure 9(b). In general, anatase shows more disorder and less recovery, but it is difficult to distinguish between rutile and brookite. This demonstrates the high statistical accuracy required for quantitative analysis and provides additional post-hoc justification of the small thermal spike methodology using in this work. 


\section{Summary}

We have performed molecular dynamics simulations of spherical thermal spikes to study radiation response in the rutile, brookite and anatase polymorphs of $\mathrm{TiO}_{2}$. Using this approach we obtain excellent statistics which enables a quantative comparison of defect generation between the polymorphs. The simulations are in excellent agreement with experiment, reproducing the observed behaviour that rutile is highly resistant to amorphization, anatase is easily amorphized and that brookite exhibits intermediate behaviour. To understand the origin of these differences we performed simulations in which the density of the crystal is altered. When the density of the polymorphs was made to be similar to one another the large differences in radiation response disappeared, an observation which confirms a prior hypothesis that volumetric strain relative to the amorphous state is the primary explanation for the differing radiation response seen in the polymorphs of $\mathrm{TiO}_{2}$. This insight that density is a critical parameter may well prove instructive for understanding radiation response in other materials systems in which chemical composition is constant and multiple phases are accessible.

[1] Zinkle S J and Kinoshita C 1997 J. Nucl. Mater. 251200

[2] Weber W J et al 1998 J. Mater. Res. 131434

[3] Lumpkin G R, Pruneda M, Rios S, Smith K L, Trachenko K, Whittle K R and Zaluzec N J 2007 J. Solid State Chem. 1801512

[4] Lumpkin G R, Smith K L, Blackford M G, Thomas B S, Whittle K R, Marks N A and Zaluzec N J 2008 Phys. Rev. B $\mathbf{7 7} 214201$

[5] Lumpkin G R, Blackford M G, Smith K L, Whittle K R, Zaluzec N J, Ryan E A and Baldo P 2010 Am. Mineralogist 95192

[6] Sickafus K E, Grimes R W, Valdez J A, Cleave A, Tang M, Ishimaru M, Corish S M, Stanek C R and Uberuaga B P 2007 Nat. Mater. 6217

[7] Sickafus K E, Valdez J A, Williams J R, Grimes R W and Hawkins H T 2002 Nucl. Instrum. Methods Phys. Res. B 191549

[8] Marks N A, Thomas B S, Smith K L and Lumpkin G R 2008 Nucl. Instrum. Methods Phys. Res. B 2662665

[9] Devanathan R 2009 Nucl. Instrum. Methods Phys Res B 2673017

[10] Devanathan R and Weber W J 2010 Nucl. Instrum. Methods Phys. Res. B 2682857

[11] Trachenko K, Dove M T, Artacho E, Todorov I T and Smith W 2006 Phys. Rev. B 73174207

[12] Santos I, Marquès L A and Pelaz L 2010 Phy. Rev. B $\mathbf{7 4} 174115$

[13] Crocombette J P 2009 Nucl. Instrum. Methods Phys. Res. B 2673152

[14] Matsui M and Akaogi M 1991 Mol. Simulat. 6239

[15] Collins D R and Smith W 1996 Technical report DL-TR-96-001, Council for the Central Laboratory of Research Councils

[16] Thomas B S, Marks N A and Begg B D 2004 Phys. Rev. B 69144122

[17] Ziegler J F, Biersack J P and Littmark U 1985 The Stopping And Range of Ions in Matter (Pergamon, New York)

[18] Smith R, Ramasawmy D and Kenny S D 2005 Nucl. Instrum. Methods Phys. Res. B 228330

[19] Smith R, Bacorisen D, Uberuaga B P, Sickafus K E, Ball J A and Grimes R W 2005 J. Phys: Condens. Matter 17875

[20] Todorov I T, Smith W, Trachenko K and Dove M T 2006 J. Mater. Chem. 161911

[21] Corrales L R, Chartier A and Devanathan R 2005 Nucl. Instrum. Methods Phys. Res. B 228 274

[22] Corrales L R 2004 Nucl. Instrum. Methods Phys. Res. B 21895

[23] Corrales L R, Weber W J, Chartier A, Meis C and Crocombette J P 2003 J. Phys.: Condens. Matter 156447

[24] Meagher E P and Lager G A 1979 Canadian Mineralogist 1777

[25] Horn M, Schwerdtfeger C F and Meagher E P 1972 Zeitschrift fur Kristallographie 136273 
[26] Gale J D 1997 J. Chem. Soc. Farad. Trans. 93629

[27] Robinson M, Marks N A and Lumpkin G R 2012 Phys. Rev. B 86134105

[28] Gupta P K 1993 J. Am. Ceram. Soc. 761088

[29] Hobbs L W, Sreeram A N, Jesurum C E and Berger B A 1996 Nucl. Instrum. Methods Phys. Res. B 11618

[30] Hoang V V 2007 J. Phys.: Condens. Matter 19416109

[31] Hanaor D A H and Sorrell C C 2011 J. Mater. Sci. 46855 